\title{
Adverse events following immunisation (AEFI) reports from the Zimbabwe expanded programme on immunisation (ZEPI): an analysis of spontaneous reports in Vigibase ${ }^{\circledast}$ from 1997 to 2017
}

\author{
Josiah Tatenda Masuka ${ }^{1,2^{*}}$ and Star Khoza ${ }^{3,4}$
}

\begin{abstract}
Background: Vaccine safety surveillance is an essential requirement in vaccination programmes. It supports signal identification, hypothesis generation, and the identification and rectification of gaps in vaccine pharmacovigilance systems. The objectives of this study were to determine the characteristics and trends of adverse events following immunisation (AEFI) and to assess the performance of the Zimbabwe Expanded Immunisation Programme safety surveillance system.
\end{abstract}

Methods: We carried out a descriptive study of passively collected vaccine-related Individual Case Safety Report (ICSR) data submitted to the World Health Organization global adverse drug reaction database (VigiBase ${ }^{\oplus}$ ) from Zimbabwe during the period 1997 to 2017. We extracted AEFI/ICSR data using VigiLyze ${ }^{\circledast}$ for analysis with respect to the demographic distribution, AEFI characteristics, reporting trends over time, ICSR timeliness and case completeness.

Results: A total of 272 vaccine-related ICSRs were included in the analyses with a median completeness score of 0.90 interquartile range, IQR (0.63; 0.90). The overall annual reporting rate was 0.58 per 100,000 vaccine doses and the AEFI reporting ratio ranged between 0 and $30.2 \mathrm{AEFI}$ reports per 100,000 surviving infants. The majority of ICSRs were male $(55.3 \% ; p$ value $=0.641)$ and the median age was $12(0-168)$ months. The majority of ICSRs were reported in children who had received measles $(n=133 ; 48.9 \%)$ and OPV/DTP-Hib-HepB $(n=107 ; 39.3 \%)$ vaccines. Of the 387 observed AEFIs, 301 (77.8\%) were systemic events and 86 (22.2\%) were local reactions. Systemic events were more frequently reported with doses containing the measles antigen $(n=190 ; 49.1 \%)$ while local events were associated with the multiple antigen OPV/DTP-Hib-HepB ( $n=62 ; 16.0 \%)$. The multiple antigen OPV/DTP-Hib-HepB was associated with higher rates for injection site abscess $(n=57)$, pyrexia $(n=27)$, diarrhea $(n=15)$, vomiting $(n=12)$, and seizures $(n=6)$. The measles antigen was associated with higher rates for rash $(n=44)$, ocular disorders $(n=26)$, pyrexia $(n=26)$, urticaria $(n=22)$, diarrhea $(n=8)$, and vomiting $(n=12)$.

Conclusions: Most of the ICSRs were associated with measles and OPV/DTP-Hib-HepB vaccines. Zimbabwe's vaccine safety surveillance system is still developing and is not yet fully functional. However, the current system provides a reference point for the monitoring of the ongoing AEFI reporting trends and characteristics.

Keywords: AEFI reporting, Vaccine safety, Pharmacovigilance, Immunization, Children

\footnotetext{
* Correspondence: josiahmasuka@gmail.com

${ }^{1}$ Harare Central Hospital, PO Box ST14, Southerton, Harare, Zimbabwe

${ }^{2}$ Department of Dermatology, Nelson R Mandela School of Medicine, Private

Bag X7, Congella, Durban 4013, South Africa

Full list of author information is available at the end of the article
}

(c) The Author(s). 2019 Open Access This article is distributed under the terms of the Creative Commons Attribution 4.0 International License (http://creativecommons.org/licenses/by/4.0/), which permits unrestricted use, distribution, and reproduction in any medium, provided you give appropriate credit to the original author(s) and the source, provide a link to the Creative Commons license, and indicate if changes were made. The Creative Commons Public Domain Dedication waiver (http://creativecommons.org/publicdomain/zero/1.0/) applies to the data made available in this article, unless otherwise stated. 


\section{Background}

The World Health Organisation (WHO) mandates the systematic collection, analysis and evaluation of medically important adverse events following immunisation (AEFI) for all immunisation programmes [1, 2]. The major goal of this immunisation safety surveillance is the "early detection and analysis of adverse events to allow for appropriate and quick responses to emerging AEFI issues in order to decrease the negative impact on the health of individuals and the immunisation programme" $[1,3]$. In addition, vaccine safety surveillance allows signal identification, hypothesis generation, and the identification and rectification of gaps in the system to strengthen the Expanded Programme on Immunisation (EPI programme) [1, 4].

Careful and continuous analysis of the post-marketing vaccine safety surveillance data provides a means to critically evaluate and communicate up-to-date information to the public on the benefit-risk profiles of individual vaccines. This helps to counter the negative perceptions on vaccination and the resultant vaccine hesitancy by improving the transparency in the immunisation programmes [5, 6]. A good example is provided by the Australia surveillance system which collates and reviews AEFI data submitted to national medicines regulator, the Therapeutic Goods Administration (TGA) annually since 2003 [7]. Through this system, Australia regularly updates its immunisation recommendations accordingly, thereby maximising the benefit-risk balance for the registered vaccines. However, in most developing countries there is limited vaccine pharmacovigilance infrastructure which subsequently reduces the capacity for continuous review of AEFI data [8].

The attendant introduction of new vaccines developed specifically for tropical diseases such as dengue fever, malaria and group A meningococcal meningitis necessitates a strengthening of the AEFI surveillance systems in these regions $[3,9]$. In addition, there is limited information on the performance, quality, responses to serious AEFI issues and the characteristics of the reported AEFIs in developing countries. The aim of the present study was to provide an initial assessment of the performance and quality of Zimbabwe's AEFI surveillance system. The study provides a detailed analysis of the characteristics of the reported AEFIs and individual case safety reports (ICSRs): the demographic distribution, seriousness, timeliness, completeness, types and/or classification of the AEFIs, and the reporting trends over time. Furthermore, we explored whether there were any annual AEFI reporting trends during the study period.

\section{Methods}

\section{Study setting}

Zimbabwe has been a full member of the WHO Programme for International Drug Monitoring (PIDM) since 1998. Full membership to the WHO PIDM requires a demonstration of technical competence in managing ICSRs by submitting at least 20 reports to the WHO global ICSR database, VigiBase ${ }^{\bullet}$. ADR reporting activities are coordinated through the Medicines Control Authority of Zimbabwe (MCAZ). MCAZ submits AEFI ICSRs collected from the national EPI vaccine safety surveillance programme to VigiBase ${ }^{\bullet}$ as provided in the national AEFI guidelines [10]. This system is designed to capture AEFI reports from the Zimbabwean birth cohort with an estimated official vaccine coverage of around 90\% for the surviving infants (using the 2017 GAVI estimates for DTP coverage as a proxy for the national immunisation coverage) [11]. The country has a relatively strong immunisation programme as indicated by a less than 10\% drop-out rate between DTP1 and DTP3 coverage [12].

AEFI surveillance and investigation is managed and coordinated by the Zimbabwean Expanded Programme of Immunisation (ZEPI) team, a division of the Ministry of Health and Child Care. All the collected AEFI ICSRs are sent to the Medicines Control Authority of Zimbabwe for further analysis, causality assessment and subsequent upload into VigiBase ${ }^{\oplus}$. The causality assessment is done using the WHO Aide Memoire tool by the national (central) expert committee which comprises of paediatricians, physicians, pharmacists, clinical pharmacologists and a vaccination nurse. Further investigations and information is provided by the ZEPI team when needed by the expert committee to finalise AEFI causality assessments [10].

\section{Study design}

We carried out a descriptive study using passively collected spontaneous AEFI surveillance data from Zimbabwe covering the period 1997 to 2017. The de-identified Individual Case Safety Reports (ICSRs) were extracted from VigiBase ${ }^{\circ}$ using its search and analysis software database known as VigiLyze $^{\curvearrowleft}$ on 2017-09-16, dataset date: 2017-09-10. ICSRs which met the following criteria were included in the analysis: an AEFI, at least one suspected vaccine included in the routine EPI, and an identifiable patient in accordance with the International Conference on Harmonisation (ICH) E2D guideline [13]. We excluded AEFI reports emanating from vaccines not used in the ZEPI programme. These included AEFI reports for adults (i.e. > 18 years), rabies and tetanus vaccines.

An AEFI was defined as any untoward medical occurrence which follows immunisation and which does not necessarily have a causal relationship with the usage of the vaccine [1]. The denominator for calculating the AEFI rates was derived by multiplying the population of children immunized by the estimated coverage. The population and vaccination coverage estimates used were obtained from the 2017 GAVI Zimbabwe country factsheet [11]. Based on 
the 2017 GAVI Zimbabwe country factsheet, we assumed a birth cohort of 537,364 children, 515,965 surviving infants, 90\% vaccination coverage, and 20 birth cohorts from 1997 to 2017 to calculate the AEFI reporting rates (per 100,000 vaccine doses). Assuming that 464,368 children of each birth cohort are vaccinated, a total population of 9,287,360 children was used as the denominator to estimate the administered vaccine doses. In cases of co-administration of two or more vaccines in an individual, we attributed the reported AEFI to the reporter suspected vaccine. The AEFI reporting ratio (per 100,000 surviving infants) as defined in the WHO's Global Vaccine Action Plan (GVAP) for vaccine safety monitoring was calculated as a product of coverage $\mathrm{X}$ population using the WHO annual estimates for surviving infants from 1997 to 2017 [14]. The number of surviving infants was obtained from the United Nations Population Division statistics and the Zimbabwe GAVI country factsheet 2017 [11, 12].

Two vaccination schedules were used in the period under review differentiated by the immunisation schedule change carried out in 2012. The vaccination schedule prior to the 2012 schedule change was as follows: at birth - BCG, at 3, 4, 5 months - OPV, DTP-Hib-HepB plus/minus pneumococcal conjugate and rotavirus vaccines; at 9 months - measles; at 18 months - OPV and DTP boosters and at 5 years OPV and DTP boosters [15]. The new schedule maintains BCG at birth, measles antigen at 9 months and the OPV and DTP boosters at 18 months, but provides for administration of OPV, DTPHib-HepB plus/minus pneumococcal conjugate and rotavirus vaccines at 6,10 and 14 weeks whilst dropping the booster doses previously given at 5 years of age. Table 1 shows the vaccination schedules used in Zimbabwe before 2012 and after 2012.

\section{Statistical analysis}

The de-duplicated, MedDRA version 20.0 coded data was exported onto a Microsoft Office Excel $^{\text {TM }}$ package (Microsoft Corporation, Redmond, WA, USA). Further analysis was done using the Statistical Package for Social Sciences (SPSS) version 22.0 (IBM Corporation, Somers, NY, USA) and Stata Version 12 (College Station, TX, USA).

Data were first analysed using descriptive statistical methods. Measures of central tendency for continuous variables were displayed as means and their corresponding standard deviations or medians and their corresponding ranges' lower and upper (Q25;Q75) values. The one way ANOVA and student's t tests were used to compare continuously distributed variables whereas categorical variables were compared using the chi-squared and/or Fischer's exact test accordingly. A descriptive time-series analysis with the $\mathrm{C}$-statistic was used to analyse the yearly AEFI reporting trends as described by Tryon, 1982 [16]. The first 8 counts were used as the baseline data whilst the subsequent 12 counts were used to analyse whether there were any reporting trends [16]. The basic question was whether there was any demonstrable trend in yearly reporting since the establishment of AEFI reporting through the MCAZ. All statistical tests were done at the $5 \%$ significance level.

\section{Ethical considerations}

Ethical exemption for the study was granted by the Medical Research Council of Zimbabwe (MRCZ Ref: $\mathrm{MRCZ/E/207).} \mathrm{The} \mathrm{ethics} \mathrm{review} \mathrm{exemption} \mathrm{was} \mathrm{granted}$ because AEFI reporting is a routine surveillance program which does not require informed consent and uses depersonalized, de-identified data.

\section{Results}

Demographic characteristics and quality of ICSR reports During the period under review, two hundred and eighty nine (289) vaccine-related ICSRs were extracted from VigiBase $^{\circ}$. This represents $10.4 \%$ of the 2777 medicine related ICSRs in the VigiBase ${ }^{\circ}$ database from Zimbabwe on the date the data was extracted. A total of 17 ICSRs were excluded from further analysis because they had

Table 1 Vaccines used for routine mandatory vaccination of children in Zimbabwe

\begin{tabular}{|c|c|c|c|}
\hline Vaccine & $\begin{array}{l}\text { Vaccination schedule } \\
\text { before } 2012 \text { (age in months) }\end{array}$ & $\begin{array}{l}\text { Vaccination schedule } \\
\text { from } 2012 \text { (age in months) }\end{array}$ & Antigen (s) \\
\hline BCG & 0 & 0 & bacillus Calmette-Guerrin \\
\hline Measles & 9 & 9 & measles, rubella \\
\hline OPV & $3,4,5$ & $6,10,14$ weeks & polio \\
\hline DTP-Hib-HepB & $3,4,5$ & $6,10,14$ weeks & $\begin{array}{l}\text { diphtheria, tetanus, whole } \\
\text { cell pertussis, hepatitis b, } \\
\text { haemophilus influenza }\end{array}$ \\
\hline Pneumococcal conjugate & & $6,10,14$ weeks & pneumococcal conjugate \\
\hline OPV booster & 18,60 & 18 & polio \\
\hline DTP booster & 18,60 & 18 & $\begin{array}{l}\text { diphtheria, tetanus, } \\
\text { whole cell pertussis }\end{array}$ \\
\hline
\end{tabular}


either a non-EPI or a non-paediatric vaccination indication as shown in Fig. 1. Therefore, a total of 272 ICSRs (263 routine EPI and 9 unclassified) were included for further analysis. Of the 272 ICSRs included in the analysis, 230 (84.6\%) were reported by nurses, 35 (12.9\%) were reported by physicians, $1(0.4 \%)$ report was submitted by a pharmacist whilst the remaining $6(2.2 \%)$ were reported by unspecified healthcare professionals.

Table 2 shows the demographic characteristics and the frequency of ICSRs across different antigens/vaccines. Overall, ICSRs were more frequently reported in males $(55.4 \%)$ than females, although there was no association between ICSRs and gender $(p=0.641)$. The overall median age was 12 months (range $0-168$ ) and the highest median age was observed in children vaccinated with measles (Median age $=72$ months; range 9-168). The average completeness score was $0.81 \pm 0.01$, whilst the median score was 0.90 (IQR: 0.63; 0.90). ICSRs which originated from unclassified vaccines $(0.61 \pm 0.04)$ had the lowest completeness score. Figure 2 shows the completeness score ranges for ICSRs for the different vaccines. For ICSR entries with complete data, the average time from the onset of an AEFI to VigiFlow entry was $1205.94 \pm 993.27$ days whilst the median time was 734.00 days (IQR: $657.75 ; 2068.00$ days). Figure 3 shows the timeliness of reporting for ICSRs for the different vaccines.

\section{Vaccines}

The majority of the 272 ICSRs could be grouped into the BCG, measles, OPV/DTP-Hib-HepB and OPV/DTP vaccination groupings. The proportions of ICSRs contributed by live attenuated vaccines (BCG and measles) and the combination doses with a mixture of inactivated, subunit and toxoid vaccines are shown in Table 2 . The majority of ICSRs were reported in children who had received measles $(n=133 ; 48.9 \%)$, and OPV/DTP-HibHepB $(n=107 ; 39.3 \%)$ vaccines. However, some of the vaccinations were unclassified $(n=9 ; 2.3 \%)$. Measles was inappropriately administered outside the scheduled age in 40 children (14.7\%).

\section{Outcomes and seriousness assessment}

About 66.9\% (259/387) of the reported AEFIs were recorded as either recovered or recovering with the remainder recorded as either an unknown outcome or death. The prevalence of serious events were calculated using the total number of ICSRs and the number of ICSRs reported per vaccine as the denominator. Of the total 272 reported ICSRs, 30 (11.0\%) were recorded as serious events. Of the serious events, 16 (5.9\%) were deaths, $10(3.7 \%)$ were life threatening, and $4(1.5 \%)$ were prolonged hospitalizations. Serious events occurred with higher frequencies in children who received OPV/DTPHib-HepB $(n=14 ; 13.1 \%)$ and measles inappropriately $(n=7 ; 17.5 \%)$. Out of the seven deaths that occurred in neonates, five were observed in children who had inappropriate (wrong age) administration of OPV/ DTP-Hib-HepB. BCG $(n=2 ; 12.5 \%)$, OPV/DTP-HibHepB $(n=9 ; 8.4 \%)$, and measles administered inappropriately $(n=3 ; 7.5 \%)$ were associated with higher frequencies of deaths compared to other vaccines $(p=0.001)$. However, no causality information was recorded to assess the relatedness of the vaccine/antigens to the deaths.

\section{Adverse event distribution}

There were more systemic AEFIs compared to local AEFIs reported during the period under review. Systemic events were more frequently reported with doses

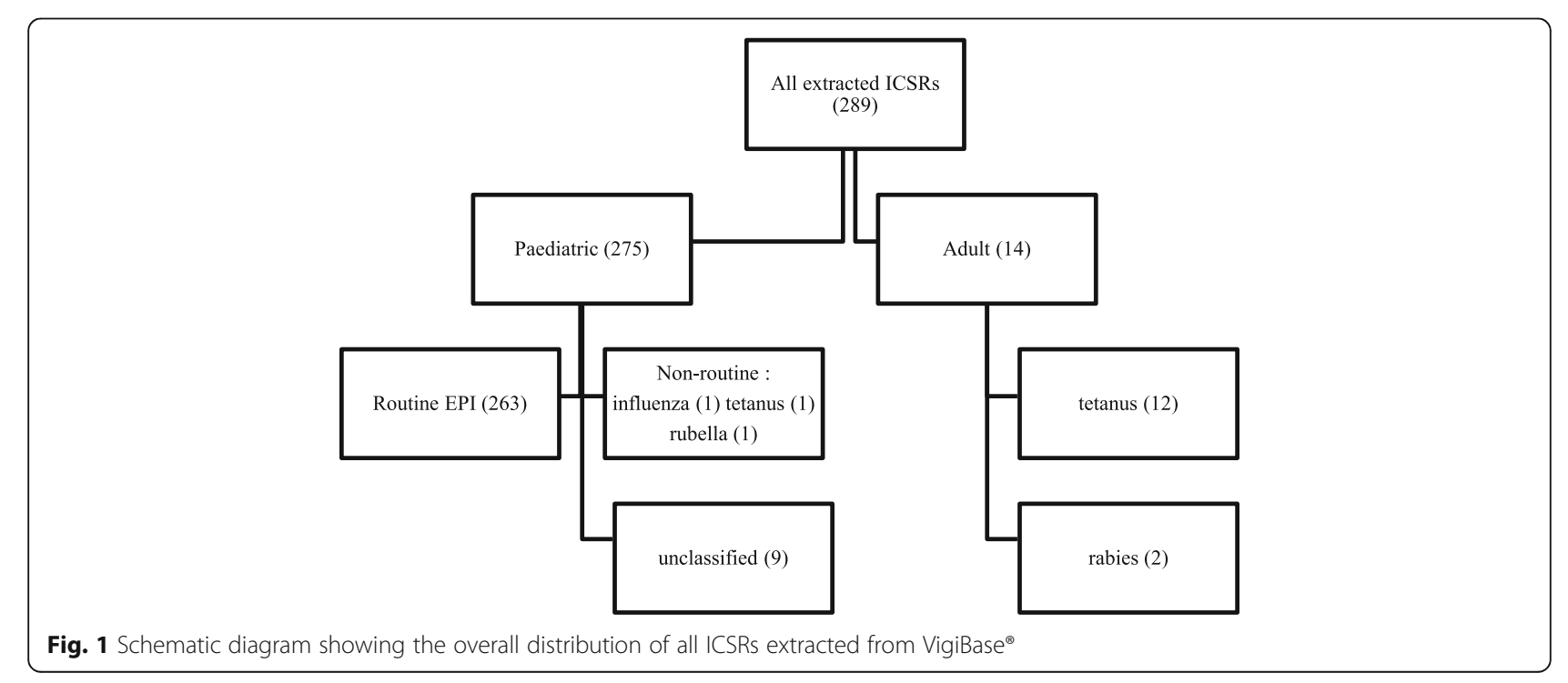


Table 2 Demographic characteristics and frequency of ICSRs per antigen or vaccine

\begin{tabular}{|c|c|c|c|c|c|c|c|c|}
\hline \multirow[t]{2}{*}{ Variable } & \multirow{2}{*}{$\begin{array}{l}\text { Total ICSRs } \\
(N=272) \\
n(\%)^{a}\end{array}$} & \multicolumn{6}{|l|}{ Vaccine } & \multirow[t]{2}{*}{$P$ value } \\
\hline & & $\begin{array}{l}\mathrm{BCG}(N=16) \\
\mathrm{n}(\%)^{\mathrm{a}}\end{array}$ & $\begin{array}{l}\text { Measles } \\
(N=93) \mathrm{n}(\%)^{\mathrm{a}}\end{array}$ & $\begin{array}{l}\text { Measles inappropriate } \\
(N=40) n(\%)^{\mathrm{a}}\end{array}$ & $\begin{array}{l}\text { OPV/DTP booster } \\
(N=7) n(\%)^{\mathrm{a}}\end{array}$ & $\begin{array}{l}\text { OPV/DTP-Hib-HepB } \\
(N=107) n(\%)^{\mathrm{a}}\end{array}$ & $\begin{array}{l}\text { Unclassified } \\
(N=9) n(\%)^{a}\end{array}$ & \\
\hline $\begin{array}{l}\text { ICSR } \\
\text { completeness } \\
\text { Mean } \pm \text { SE }\end{array}$ & $0.81 \pm 0.01$ & $0.79 \pm 0.04$ & $0.82 \pm 0.02$ & $0.87 \pm 0.01$ & $0.82 \pm 0.05$ & $0.79 \pm 0.02$ & $0.61 \pm 0.04$ & $<0.0001$ \\
\hline \multicolumn{9}{|l|}{ Gender } \\
\hline Male & $150(55.3)$ & $7(43.7)$ & $55(59.8)$ & $22(55.0)$ & $4(57.1)$ & $59(55.1)$ & $3(33.3)$ & 0.641 \\
\hline Female & $121(44.7)$ & $9(56.3)$ & $37(40.2)$ & $18(45.0)$ & $3(42.9)$ & $48(44.9)$ & $6(66.7)$ & \\
\hline $\begin{array}{l}\text { Male: female } \\
\text { Ratio }\end{array}$ & 1.24 & 0.78 & 1.49 & 1.22 & 1.33 & 1.23 & 0.50 & \\
\hline \multicolumn{9}{|l|}{ Age (months) } \\
\hline Median & 12 & 2.5 & 72 & 17 & 60 & 5 & 5 & $<0.0001$ \\
\hline Range & $0-168$ & $0-11$ & $9-168$ & $4-48$ & $17-60$ & $0-12$ & $3-6$ & \\
\hline Number of AEFIs & $387(100.0)$ & $20(5.2)$ & 136(35.1) & $60(15.5)$ & $10(2.6)$ & $152(39.3)$ & $9(2.3)$ & $<0.0001$ \\
\hline AEFI/ICSR ratio & 1.42 & 1.25 & 1.46 & 1.50 & 1.43 & 1.42 & 1.00 & \\
\hline \multicolumn{9}{|l|}{ Severity of ICSR } \\
\hline Serious & $30(11.0)$ & $2(12.5)$ & $6(6.5)$ & $7(17.5)$ & $1(14.3)$ & 14(13.1) & $\mathrm{O}(0.0)$ & 0.371 \\
\hline Not serious & $242(89.0)$ & $14(87.5)$ & $87(93.5)$ & $33(82.5)$ & $6(85.7)$ & 93(86.9) & $9(100.0)$ & \\
\hline \multicolumn{9}{|l|}{ Seriousness of ICSR } \\
\hline $\begin{array}{l}\text { Prolonged } \\
\text { Hospitalisation }\end{array}$ & $4(1.5)$ & $0(0.0)$ & $1(1.1)$ & $0(0.0)$ & $0(0.0)$ & $3(2.8)$ & $0(0.0)$ & 0.051 \\
\hline Life Threatening & $10(3.7)$ & $0(0.0)$ & $3(3.2)$ & $4(10.0)$ & $1(14.3)$ & $2(1.9)$ & $0(0.0)$ & 0.156 \\
\hline Death & $16(5.9)$ & $2(12.5)$ & $2(2.2)$ & $3(7.5)$ & $0(0.0)$ & $9(8.4)$ & $0(0.0)$ & 0.001 \\
\hline
\end{tabular}

Key: ${ }^{a}$ column percentages; ${ }^{b}$ row percentages

containing the measles antigen $(n=190 ; 49.1 \%)$ while local events were associated with the multiple antigen OPV/DTP-Hib-HepB vaccine $(n=62 ; 16.0 \%)$. The most common systemic AEFIs were pyrexia $(n=61 ; 15.8 \%)$ and rash $(n=49 ; 12.7 \%)$. The most frequently reported local AEFI was the injection site abscess $(n=75 ; 19.4 \%)$. The multiple antigen OPV/DTP-Hib-HepB vaccine was associated with higher rates of injection site abscess $(n=$ $57 ; 37.5 \%)$, pyrexia $(n=27 ; 17.8 \%)$, diarrhoea $(n=15$; $9.9 \%)$, vomiting ( $n=12 ; 7.9 \%)$, and seizures $(n=6 ; 3.9 \%)$.

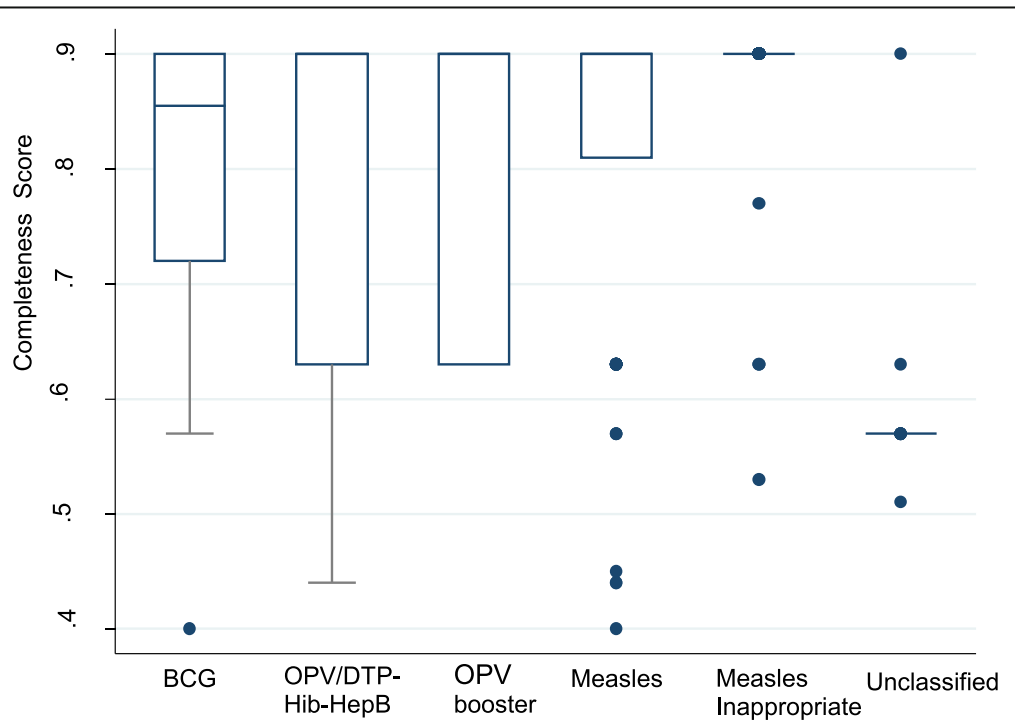

Fig. 2 Box and Whisker plots ICSR reporting completeness scores for each vaccine/antigen 


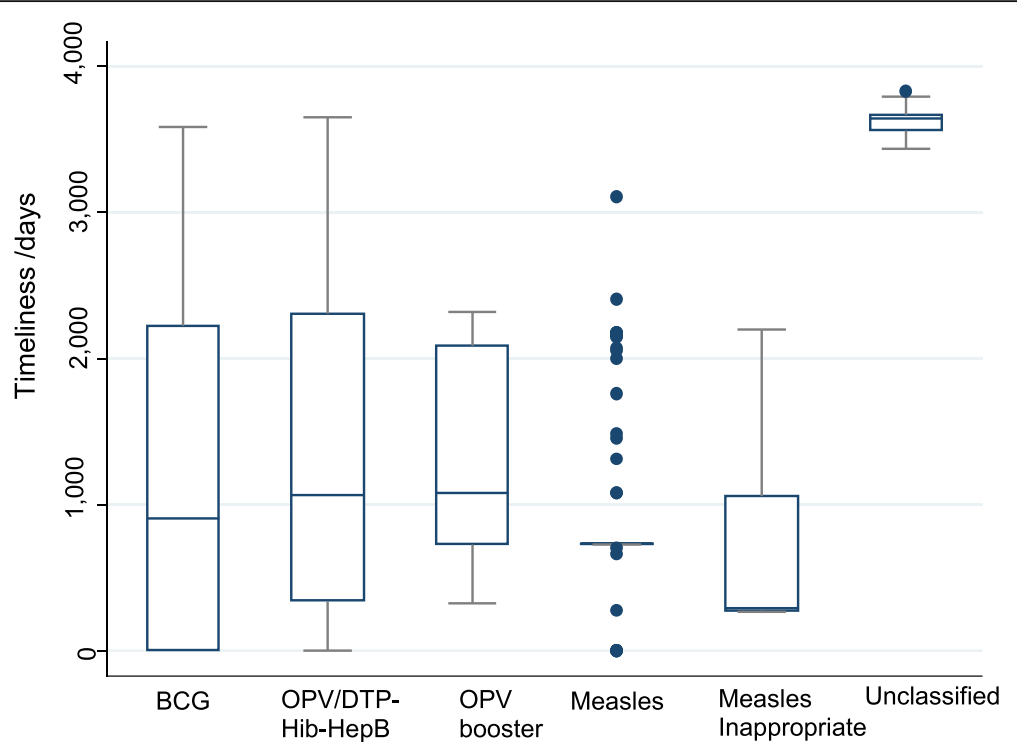

Fig. 3 Box and Whisker plots ICSR reporting timeliness for each vaccine/antigen

The measles antigen was associated with higher rates of rash $(n=44 ; 22.4 \%)$, ocular disorders $(n=17 ; 8.7 \%)$, pyrexia $(n=26 ; 13.3 \%)$, urticaria $(n=22 ; 11.2 \%)$, diarrhoea ( $n=8 ; 4.1 \%)$, vomiting $(n=12 ; 6.1 \%)$, hypersensitivity reactions $(n=4 ; 2.0 \%)$. Table 3 shows the distribution of AEFIs across different antigens/vaccines.

\section{Reporting trends}

Table 4 shows the AEFI reporting rates for the individual vaccines. The overall AEFI reporting rate was 0.58 per 100,000 vaccine doses and the AEFI/ICSR ratio was 1.42. Measles had the highest AEFI reporting rate of 2.11 per 100,000 followed by OPV/DTP-Hib-HepB at 0.54 per 100,000 vaccine doses. The highest AEFI/ICSR ratio was observed when measles was administered inappropriately (1.50) and the lowest ratio was observed with BCG vaccine (1.25). From 1997 to 2010, there was a general increase in the number of ICSRs. The highest annual count of ICSRs was observed in $2010 \quad(n=74$; 27.2\%). The annual counts of the ICSRs reported during the period 1997 to 2017 are shown in Fig. 4. The annual AEFI reporting ratio ranged between 0 and 30.2 per 100, 000 surviving infants. Table 5 shows the annual number of ICSRs, AEFIs, and AEFI reporting ratios from 1997 to 2017. There was a significant increase in the reporting of ICSRs during the baseline and the reporting periods $(p<0.05)$.

\section{Discussion}

The main purpose of this descriptive study was to provide a detailed analysis of the characteristics, quality and reporting trends of AEFIs captured from the Zimbabwe Expanded Immunisation Programme (ZEPI) as recommended by the WHO's Global Manual in the surveillance of AEFI [1]. Data extracted from the WHO's global pharmacovigilance database, VigiBase $^{\bullet}$, for Zimbabwe for the period 1997 to 2017 was used as it represented the entire AEFI dataset submitted by the national pharmacovigilance centre. We found an overall AEFI reporting rate of 0.58 per 100,000 vaccine doses and the annual AEFI reporting ratio ranged between 0 and 30.2 AEFI reports per 100,000 surviving infants. There was a steady increase in the AEFI reporting trends over time. The peak in AEFI reporting observed in 2009 could have been due to enhanced vaccine safety awareness associated with the pandemic H1N1 influenza virus vaccination campaign during this period as also observed by $\mathrm{Hu}$ et al. in Zhenjiang, China [17]. The decrease in the reporting rates observed from 2014 could be due to pending collection and uploading of the ICSRs into VigiBase $^{\circ}$ by the national pharmacovigilance centre. The widely varying annual AEFI reporting ratio resultant from the varying AEFI reporting trends indicates that Zimbabwe's vaccine safety system is still developing. A functional AEFI reporting system should have a minimal annual reporting ratio of 10 AEFI reports per 100,000 surviving infants as indicated in the WHO's Global Vaccine Action Plan for vaccine safety monitoring [14].

The observed $11.0 \%$ of AEFIs classified as serious is consistent with findings from passive surveillance 
Table 3 Distribution of AEFls across antigens/vaccines

\begin{tabular}{|c|c|c|c|c|c|c|c|c|}
\hline \multirow[t]{2}{*}{ Variable } & \multirow{2}{*}{$\begin{array}{l}\text { Total } \\
\text { number } \\
\text { of AEFIs } \\
(\mathrm{N}=387) \\
\mathrm{n}(\%)^{\mathrm{a}}\end{array}$} & \multicolumn{6}{|l|}{ Vaccine } & \multirow[t]{2}{*}{$P$ value } \\
\hline & & $\begin{array}{l}\text { BCG }(N= \\
20) n(\%)^{a}\end{array}$ & $\begin{array}{l}\text { Measles }(N= \\
136) \mathrm{n}(\%)^{\mathrm{a}}\end{array}$ & $\begin{array}{l}\text { Measles } \\
\text { inappropriate }(N= \\
60) n(\%)^{a}\end{array}$ & $\begin{array}{l}\text { OPV/DTP booster } \\
(N=10) n(\%)^{a}\end{array}$ & $\begin{array}{l}\text { OPV/DTP-Hib-HepB } \\
(N=152) n(\%)^{\mathrm{a}}\end{array}$ & $\begin{array}{l}\text { Unclassified } \\
(N=9) n(\%)^{a}\end{array}$ & \\
\hline \multicolumn{9}{|l|}{ Type of AEFI } \\
\hline Systemic & $301(77.8)$ & $13(65.0)$ & 130(95.6) & $60(100.0)$ & $6(60.0)$ & $90(59.2)$ & $2(22.2)$ & $<0.0001$ \\
\hline Local & $86(22.2)$ & $7(35.0)$ & $6(4.4)$ & $0(0.0)$ & $4(40.0)$ & $62(40.8)$ & $7(77.8)$ & \\
\hline \multicolumn{9}{|l|}{ Systemic AEFIs } \\
\hline Cough & $5(1.3)$ & 0 & 3 & 1 & 0 & 1 & 0 & 0.146 \\
\hline Diarrhoea & $23(5.9)$ & 0 & 3 & 5 & 0 & 15 & 0 & $<0.0001$ \\
\hline Dyspnoea & $4(1.0)$ & 0 & 1 & 0 & 0 & 3 & 0 & 0.051 \\
\hline Hypersensitivity & $4(1.0)$ & 0 & 4 & 0 & 0 & 0 & 0 & 0.001 \\
\hline $\begin{array}{l}\text { Occular } \\
\text { Disorders }\end{array}$ & $17(4.4)$ & 0 & 14 & 3 & 0 & 0 & 0 & $<0.0001$ \\
\hline Paralysis & $4(1.0)$ & 0 & 1 & 1 & 1 & 1 & 0 & 0.841 \\
\hline Pyrexia & 61(15.8) & 4 & 13 & 13 & 3 & 27 & 1 & $<0.0001$ \\
\hline Seizure & $12(3.1)$ & 0 & 2 & 4 & 0 & 6 & 0 & 0.007 \\
\hline Vomiting & $25(6.5)$ & 0 & 4 & 8 & 0 & 12 & 1 & $<0.0001$ \\
\hline Pruritus & $11(2.8)$ & 0 & 9 & 2 & 0 & 0 & 0 & $<0.0001$ \\
\hline Rash & 49(12.7) & 1 & 33 & 11 & 1 & 3 & 0 & $<0.0001$ \\
\hline Urticaria & $23(5.9)$ & 1 & 19 & 3 & 0 & 0 & 0 & $<0.0001$ \\
\hline Other & $63(16.3)$ & 7 & 24 & 9 & 1 & 22 & 0 & \\
\hline \multicolumn{9}{|l|}{ Local AEFIs } \\
\hline $\begin{array}{l}\text { Injection site } \\
\text { Abscess }\end{array}$ & 75 (19.4) & 7 & 3 & 0 & 3 & 57 & 5 & $<0.0001$ \\
\hline $\begin{array}{l}\text { Injection site } \\
\text { Swelling }\end{array}$ & $5(1.3)$ & 0 & 0 & 0 & 1 & 3 & 1 & 0.146 \\
\hline Other & $6(1.6)$ & 0 & 3 & 0 & 0 & 2 & 1 & 0.156 \\
\hline
\end{tabular}

Key: ${ }^{a}$ column percentages

systems observed in Australia (11\%), the United States (14.2\%) and Germany (19\%), but it differs markedly from the findings in Zhejiang province, China (1\%) and Croatia (3\%) [17-22]. These differences may be due to the inherent differences in the reporting practices of the different countries [17]. Similar to the observations made by Zhou et al. in China, pyrexia (15.8\%) was the most frequently reported AEFI and this commonly followed receipt of a dose of pertussis-containing vaccines (DTP) than for other vaccines [23]. However, in the absence of causality assessment, we are not able to make similar conclusions concerning neonatal deaths which occurred near the dates of vaccination. In a country with a high infant and childhood mortality, these

Table 4 Number and rates of AEFls per vaccine/antigen from 1997 to 2017

\begin{tabular}{lllll}
\hline $\begin{array}{l}\text { Vaccine/Naccine } \\
\text { combination }\end{array}$ & $\begin{array}{l}\text { Number of doses } \\
\text { per birth cohort }\end{array}$ & Number of AEFIs & $\begin{array}{l}\text { Number of vaccine } \\
\text { doses (million) }\end{array}$ & $\begin{array}{l}\text { Rate per 100,000 } \\
\text { vaccine doses }\end{array}$ \\
\hline BCG & 1 & 20 & 9.3 & 0.21 \\
Measles & 1 & $196^{\mathrm{b}}$ & 9.3 & 2.11 \\
OPV/DTP-Hib-HepB & 3 & 152 & 27.9 & 0.54 \\
OPV/DTP booster & 2 & 10 & 18.6 & 0.05 \\
Total & & $378^{\mathrm{c}}$ & 65.1 & $0.58^{\mathrm{d}}$ \\
\hline
\end{tabular}

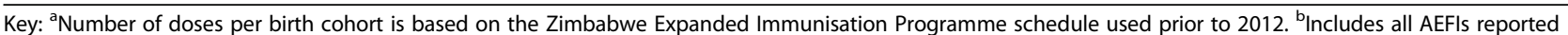
with measles regardless of the age at which the vaccine was administered. ${ }^{\mathrm{c} E x c l u d e s} \mathrm{AEFls}$ reported for unclassified vaccines. ${ }^{\mathrm{d}} \mathrm{Average}$ reporting rate per 100,000 vaccine doses 


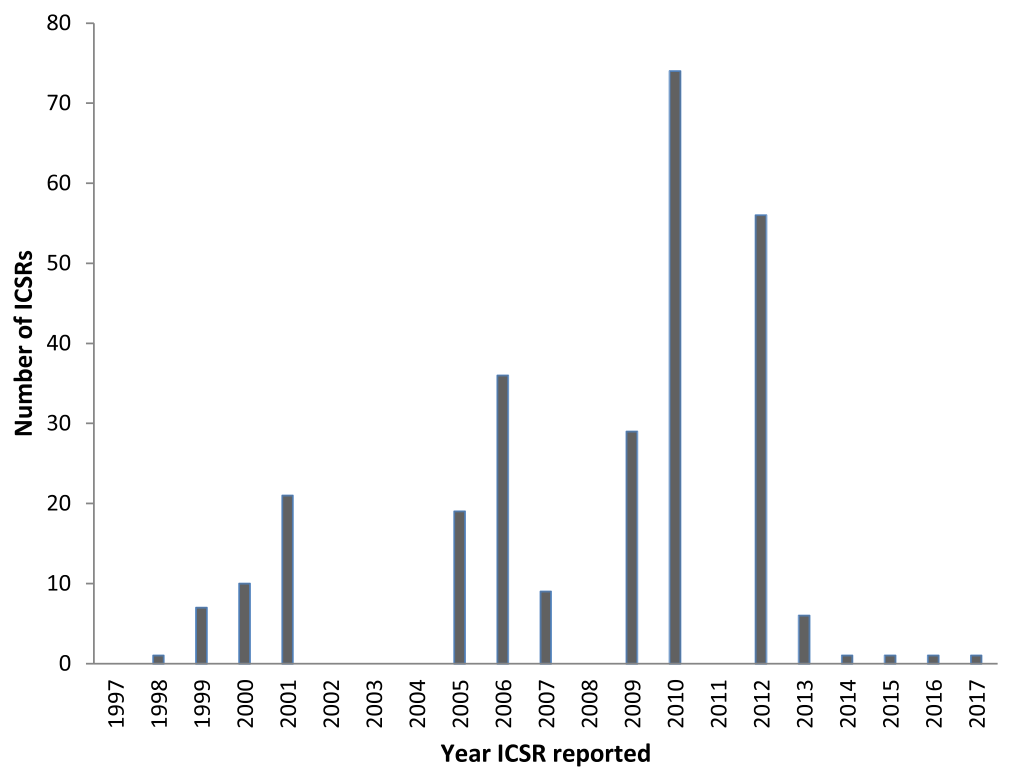

Fig. 4 Annual ICSR reporting trends for the period 1997 to 2017

Table 5 Number of ICSRs and annual AEFI ratios from 1997 to 2017

\begin{tabular}{|c|c|c|c|c|}
\hline Reporting year & Number of ICSRs & Number of AEFIs & $\begin{array}{l}\text { Number of } \\
\text { surviving infants }\end{array}$ & $\begin{array}{l}\text { Annual AEFI ratio per } \\
100,000 \text { surviving infants }\end{array}$ \\
\hline 1997 & 0 & 0 & 426,000 & 0 \\
\hline 1998 & 1 & 1 & 427,000 & 0.2 \\
\hline 1999 & 7 & 12 & 433,000 & 2.8 \\
\hline 2000 & 10 & 12 & 364,000 & 3.3 \\
\hline 2001 & 21 & 28 & 362,000 & 7.7 \\
\hline 2002 & 0 & 0 & 361,000 & 0 \\
\hline 2003 & 0 & 0 & 360,000 & 0 \\
\hline 2004 & 0 & 0 & 360,000 & 0 \\
\hline 2005 & 19 & 23 & 351,000 & 6.5 \\
\hline 2006 & 36 & 53 & 352,000 & 15.5 \\
\hline 2007 & 9 & 10 & 354,000 & 2.8 \\
\hline 2008 & 0 & 0 & 356,000 & 0 \\
\hline 2009 & 29 & 44 & 359,000 & 12.3 \\
\hline 2010 & 74 & 107 & 354,000 & 30.2 \\
\hline 2011 & 0 & 0 & 358,000 & 0 \\
\hline 2012 & 56 & 84 & 501,000 & 16.8 \\
\hline 2013 & 6 & 7 & 507,000 & 1.4 \\
\hline 2014 & 1 & 2 & 511,000 & 0.4 \\
\hline 2015 & 1 & 1 & 512,000 & 0.2 \\
\hline 2016 & 1 & 1 & 512,000 & 0.2 \\
\hline 2017 & 1 & 1 & 515,000 & 0.2 \\
\hline Total ${ }^{a}$ & $271^{a}$ & $385^{b}$ & & \\
\hline
\end{tabular}

${ }^{a}$ Excludes one ICSR which had missing information on the year the report was made

${ }^{b}$ Excludes two AEFIs from the ICSR with missing information on the year the report was made 
could be coincidental deaths. The deaths could also be attributed to other conditions associated with high infant and childhood mortality and this necessitates robust investigations and causality assessment to rule out other causes [24]. The high overall and vaccine specific male to female reporting ratios are similar to the findings in Australia in children below 12 months of age for AEFIs and below 5 years of age for other medicines [21, 25]. However, this observation differs from the findings in a study by Harris et al. which concluded that the relative reporting rate for AEFIs tends to be equal in children below 11 years of age [26]. The reasons for the AEFI preponderance in infant males are not clear.

The administration of multiple antigens or vaccines tends to produce a widening and lowering of the ICSR completeness score ranges. However, the AEFI completeness scores were markedly high compared to the global average of 0.41 observed within VigiBase ${ }^{\bullet}[27,28]$. The median time interval between the date of onset of an ADR and the date of reporting to VigiBase ${ }^{\circ}$ of 734.00 days $(\approx 2.01$ years $)$ is comparable to the 2.40 years observed in a review of global ICSR data $[29,30]$. However, the lack of follow-up data prevents a full assessment of the quality of the AEFI data. Furthermore, the zero annual reporting observed for some of the years under review suggests under-reporting as vaccines are regularly and consistently administered by the national EPI programme. Under-reporting in Zimbabwe is also highlighted by the rather low overall AEFI reporting ratio of 0.58 AEFIs per 100,000 doses. Although, this AEFI reporting rate is comparable to the 2.7 AEFIs per 100,000 doses observed in Switzerland, it [31] is lower than the Australian overall annual AEFI reporting ratio of 14.6 AEFIs per 100,000 doses in children under the age of 7 years for all scheduled vaccines [32] and the 12.4 AEFIs per 100,000 doses reported in the Spanish passive AEFI surveillance system [33]. The underdevelopment of the passive AEFI surveillance system in Zimbabwe may explain the low AEFI reporting rates compared to other AEFI surveillance systems. Furthermore, inadequate healthcare worker training on AEFI surveillance, limited coordination of AEFI surveillance activities, insufficient reporting resources and competing work priorities may also adversely affect the AEFI reporting trends in Zimbabwe [14, 34]. It should also be noted that all the AEFI reporting rates observed in Australia, Spain, Switzerland, and Zimbabwe are way lower than the global average rate of 549 AEFIs per 100,000 surviving infants reported in 2015. This global average was largely driven by the markedly high Eastern Mediterranean region's AEFI report submissions [14]. The observed AEFI reporting variations may be explained by regional differences in immunization program funding, population demographics, and AEFI reporting requirements and case definitions [35]. In addition, passive AEFI surveillance rates can be influenced by active vaccine surveillance programs and enhanced vaccine safety awareness campaigns.

Several strategies can be employed to strengthen the AEFI surveillance system in Zimbabwe. Improving healthcare professionals' awareness of vaccine safety reporting and providing appropriate and timely feedback to reporters of AEFIs may increase the quality and the numbers of reported AEFIs [36]. In addition, complementary increases in internal funding and resources such as fully functional, identifiable sentinel AEFI reporting sites, the linkage of AEFI data with immunisation registers, and the use of national vaccine safety experts may also enhance patient follow-up, AEFI investigations and the timeliness and completeness of ICSRs [36]. Furthermore, the use of participant-centred active surveillance of AEFIs can be utilised instead of passive surveillance as it is sustainable, relatively affordable and provides timely signal detection opportunities especially for post-marketing vaccine pharmacovigilance [37]. Given the shift towards vaccine development for tropical and other health challenges in resource limited settings, active, participant-centred safety surveillance may offer a unique solution to provide complete, timely and useful AEFI data. Furthermore, pharmacovigilance information documented within the UMC VigiBase ${ }^{\circ}$ system is important for monitoring vaccine safety signals [38]. Therefore, the utility of the VigiBase ${ }^{\circ}$ data can be increased by the addition of causality assessments and reporter assessed cause-specific categorisation of AEFIs during data entry. This will provide sufficient information for the comprehensive analysis AEFI safety data. In addition, cause-specific categorisation will enable the differentiation of coincidental events from vaccine reactions especially for events such as death whose occurrence and miscommunication with the community may disrupt the EPI programme [24].

This study has several limitations that should be borne in mind when interpreting these findings. First, the passive AEFI surveillance system has inherent limitations which include under-reporting of AEFI reports, inadequate training of healthcare workers in basic AEFI surveillance, variability in the accuracy and completeness of AEFI reports, and inconsistency in the application of case definitions for reportable AEFIs. Second, the AEFI rates were calculated using the birth cohort as an estimate of the number of children who were vaccinated. Therefore, the unavailability of the accurate denominator data (number of doses administered to patients) makes the AEFI rates less accurate. Although using the population of a birth cohort can lead to less accurate AEFI rates, this approach can serve as a good indicator of the performance of the AEFI surveillance system over time. Third, the lack of data on causality assessments in VigiBase $^{\circ}$ makes it difficult to make any conclusions regarding potential causal relationships between vaccines 
and specific AEFIs. Furthermore, passive AEFI surveillance systems have an inherent limitation that the observed temporal relationships between immunizations and AEFI cases do not necessarily mean that they are causal. Fourth, this study is based on AEFIs submitted to the UMC VigiBase ${ }^{\bullet}$ database after verification for validity by the Medicines Control Authority of Zimbabwe. AEFIs with incomplete or inconsistent information submitted to MCAZ are returned to the healthcare institutions for additional data. This censoring of ICSR data has the potential of reducing the number of reported ICSRs and inflating the quality of analysed ICSRs. In addition, the de-duplication algorithm in VigiBase ${ }^{\bullet}$, VigiMatch ${ }^{\circ}$, further censors the available reports with possible misrepresentation of the reporting quality and the number of ICSRs [39]. This presents challenges to the validity of analyses done using the data from VigiBase ${ }^{\oplus}$ by most developing vaccine safety reporting systems [39]. Therefore, the results of this study may underestimate the occurrence of AEFIs. Lastly, the limited follow-up data, especially for serious AEFIs, reduced our ability to fully assess the performance of the reporting system and the efficiency of assessing the circumstances around the serious adverse events. In addition, the lack of identifiable reporting health institutions reduced our ability to analyse whether there were any reporting differences between sites. Despite these limitations, this study clearly shows the performance of AEFI surveillance system in Zimbabwe.

\section{Conclusions}

Most of the ICSRs were associated with measles and OPV/DTP-Hib-HepB vaccines while the majority of the AEFIs were systemic events. The overall AEFI reporting rate was low suggesting under-reporting of AEFIs within the vaccine safety surveillance system. Zimbabwe's vaccine safety surveillance system is still developing and is not yet fully functional. However, the current system provides a reference point for the monitoring of the ongoing AEFI reporting trends and characteristics. This interim analysis provides areas of the vaccine safety surveillance that need improvement such as the recording of causality assessments, cause-specific categories of AEFIs and the need to indicate the reporting centres to derive maximum benefit from future analyses. In addition, adequate healthcare worker training on AEFI surveillance, improved coordination of AEFI surveillance activities, and improved reporting resources will strengthen the vaccine safety surveillance system.

\footnotetext{
Abbreviations

AEFI: Adverse Events Following Immunisation; CIOMS: Council for International Organizations of Medical Sciences; EPI: Expanded Programme on Immunisation; GAVI: Global Alliance for Vaccines and Immunisation; GVAP : Global Vaccine Action Plan; ICSR: Individual Case Safety Reports; MCAZ: Medicines Control Authority of Zimbabwe; PIDM: Programme for International Drug Monitoring; WHO: World Health Organization
}

\section{Acknowledgements}

Data from the WHO Collaborating Centre for International Drug Monitoring were used. The information is not homogeneous at least with respect to origin or likelihood that the pharmaceutical product caused the adverse reaction. The information in this paper does not represent the opinion of the World Health Organization. The authors are indebted to the Medicines Control Authority of Zimbabwe for contributing data to the WHO International Drug Monitoring Programme. We are also grateful to Dr. Didier Bomene Nzolo for assistance in the downloading of the AEFI data from the Vigilyze.

\section{Author contributions}

JM was involved in the conceptualisation, data analysis, interpretation, drafting and finalisation of the manuscript. SK was involved in data analysis, interpretation, revision and finalisation of the manuscript. Both authors read and approved the final version of the manuscript.

\section{Funding}

This research received no specific grant from any funding agency in the public, commercial, or not-for-profit sectors.

\section{Availability of data and materials}

Data from the WHO Collaborating Centre for International Drug Monitoring were used. The datasets used and/or analysed during the current study are available from the corresponding author on reasonable request. However, the authors will abide to the restrictions imposed by WHO policy and the Uppsala Monitoring Centre (UMC) guidelines on sharing the datasets.

\section{Ethics approval and consent to participate}

No ethical approval was required because the study used publicly available, anonymized data in accordance with the guideline 2 of CIOMS and WHO for epidemiological studies.

\section{Consent for publication}

Not applicable

\section{Competing interests}

The authors declare that they have no competing interests.

\section{Author details}

${ }^{1}$ Harare Central Hospital, PO Box ST14, Southerton, Harare, Zimbabwe. ${ }^{2}$ Department of Dermatology, Nelson R Mandela School of Medicine, Private Bag X7, Congella, Durban 4013, South Africa. ${ }^{3}$ Department of Clinical Pharmacology, College of Health Sciences, University of Zimbabwe, PO Box A178, Avondale, Harare, Zimbabwe. ${ }^{4}$ Discipline of Pharmacology and Clinical Pharmacy, School of Pharmacy, Faculty of Natural Sciences, University of the Western Cape, Private Bag X17, Bellville 7535, South Africa.

Received: 8 April 2019 Accepted: 13 August 2019

Published online: 27 August 2019

\section{References}

1. Global manual on surveillance of adverse events following immunization. https://www.who.int/vaccine_safety/publications/Global_Manual_on_ Surveillance_of_AEFI.pdf?ua=1. Accessed 10 Jan 2019.

2. World Health Organisation. Global vaccine safety. https:/www.who.int/ vaccine_safety/initiative/detection/en/. Accessed 31 July 2018.

3. Izurieta HZP, Bonhoeffer J, Chen R, Sankohg O, Laserson K, Sturkenboom M, Loucq C, Weibel D, Dodd C, et al. Roadmap for the international collaborative epidemiologic monitoring of safety and effectiveness of new high priority vaccines. Vaccine. 2013;31(35):3623-7.

4. Shimabukuro TNM, Martin D, DeStefano F. Safety monitoring in the vaccine adverse event reporting system (VAERS). Vaccine. 2015;33(36):4398-405.

5. Di Pasquale ABP, Garçon N, Stanberry LR, El-Hodhod M, Tavares Da Silva F. Vaccine safety evaluation: practical aspects in assessing benefits and risks. Vaccine. 2016;34(52):6672-80

6. Kumar DCR, Mathur M, Samdariya S, Kapoor N. Vaccine hesitancy: understanding better to address better. Isr J Health Policy Res. 2016;5(1):2.

7. Mahajan DCJ, Dey A, Macartney K, Menzies R. Annual report: surveillance of adverse events following immunisation in Australia, 2011. Commun Dis Intell Q Rep. 2012;36(4):E315-32. 
8. Cunha MPL, Dórea JG, Marques RC, Leão RS. Vaccine adverse events reported during the first ten years (1998-2008) after introduction in the state of Rondonia, Brazil. Biomed Res Int. 2013;853083:6. https://doi.org/1 0.1155/2013/853083.

9. Amarasinghe ABS, Bonhoeffer J, Carvalho S, Dodoo A, Eskola J, Larson H, Shin S, Olsson S, Balakrishnan M, et al. Effective vaccine safety systems in all countries: a challenge for more equitable access to immunization. Vaccine. 2013;31(S2):B108-14

10. Medicines Control Authority of Zimbabwe. Adverse events following immunization surveillance guidelines. In: Trials PaC. 3rd ed. Harare; 2017.

11. Medicines Control Authority of Zimbabwe. Adverse events following immunization surveillance guidelines. https://www.mcaz.co.zw/index.php/ downloads/category/15-guidelines. Accessed 20 May 2018.

12. WHO vaccine-preventable diseases: monitoring system. 2018 global summary. http://apps.who.int/immunization_monitoring/globalsummary/ countries?countrycriteria\%5Bcountry\%5D\%5B\%5D=ZWE\&commit=OK. Accessed 20 May 2018.

13. ICH. Post-approval safety data management: definitions and standards for expedited reporting E2D. In: ICH; 2003. p. 5.

14. Lei JBM, Gidudu J, Zuber P. Use of a new global indicator for vaccine safey surveillance and trends in advesre events following immunization reporting 2000-2015. Vaccine. 2018;36(12):1577-82.

15. Ministry of Health and Child Welfare. Essentual medicines list and standard treatment guidelines for Zimbabwe. 6th Edition. 2011.

16. Tryon W. A simplified time-series analysis for evaluating treatment interventions. J Appl Behav Anal. 1982;15(3):423-9.

17. Hu YLQ, Lin L, Chen E, Chen Y, Qi X. Surveillance for adverse events following immunization from 2008 to 2011 in Zhejiang Province, China. Clin Vaccine Immunol. 2013;20(2):211-7.

18. Braun MES. Descriptive epidemiology of adverse events after immunization: reports to the vaccine adverse event reporting system (VAERS), 1991-1994. J Pediatr. 1997;131(4):529-35.

19. Zhou WPV, Iskander J, English-Bullard R, Ball R, Wise R, Haber P, Pless R, Mootrey G, Ellenberg $S$. Surveillance for safety after immunization: vaccine adverse event reporting system (VAERS)--United States, 1991-2001. MMWR Surveill Summ. 2003;52(1):1-24.

20. Keller-Stanislawski BHN, Meyer C. Adverse events following immunisation in Germany from 1.1.2001 to 31.12.2003. Bundesgesundheitsbl Gesundheitsforsch Gesundheitsschutz. 2004;47(12):1151-64.

21. Lawrence GAP, Boyd I, McIntyre P, Gold M. Annual report on surveillance of adverse events following immunisation in Australia, 2006. Commun Dis Intell Q Rep. 2007:31(3):269-82.

22. Danova JKA, Celko A. Active surveillance study of adverse events following immunisation of children in the Czech Republic. BMC Public Health. 2017; 17(1):167.

23. Zhang LPS, Axelsson I, Halperin S. Acellular vaccines for preventing whooping cough in children. Cochrane Database Syst Rev. 2014;9:CD0014.

24. Gold MBM, Amarasinghe A, MacDonald N. An approach to death as an adverse event following immunization. Vaccine. 2016;34(2):212-7.

25. Castellana ECM, Cattel F. Gender differences and pharmacovigilance: analysis in the Italian population. Ita J Gender-Specific Med. 2018;4(1):27-33.

26. Harris TNJ, Fediurek J, Deeks S. Assessment of sex-specific differences in adverse events following immunization reporting in Ontario, 2012-15. Vaccine. 2017;35(19):2600-4.

27. Bervall TNG, Lindquist M. vigiGrade: a tool to identify welldocumentedindividual case reports and highlight systematic data quality issues. Drug Saf. 2014;37(1):65-77.

28. Kalaiselvan VKR, Thota P, Tripathi A, Singh G. Status of documentation grading and completenesss score for Indian individual case safety reports. Indian J Pharm. 2015;47(3):325-7.

29. Letourneau MWG, Walop W, Duclos P. Improving global monitoring of vaccine safety: a quantitative analysis of adverse event reports in the WHO adverse reactions database. Vaccine. 2008;26(9):1185-94.

30. Aagaard L, Strandell J, Melskens L, Petersen PS, Holme Hansen E. Global patterns of adverse drug reactions over a decade: analyses of spontaneous reports to VigiBase. Drug Saf. 2012;35(12):1171-82.

31. Schumacher ZBC, Heininger U. Surveillance for adverse events following immunization (AEFI) in Switzerland-1991- 2001. Vaccine. 2010;28:4059-64.

32. Lawrence GMR, Burgess M, Mclntyre P, Wood N, Boyd I, Purcell P, Isaacs D. Surveillance of adverse events following immunisation: Austraila 2000 2002. Commun Dis Intell Q Rep. 2003;27(3):307.
33. Alguacil-Ramos AM-TJ, Garrigues-Pelufo T, Portero-Alonso A, Diez-Domingo J. E. Pastor-Villalba E, Lluch-Rodrigo J. surveillance for adverse events following immunization (AEFI) for 7 years using a computerised vaccination system. Public Health. 2016:135:66-74.

34. Mutata CTC, Juru T, Shambira G, Gombe T, Nsubuga P, Tshimanga M. Evaluation of the adverse events following immunization surveillance system in Guruve district, Mashonaland Central 2017. Pan Afr Med J. 2018;31:202.

35. Annual Report on Vaccine Safety in Ontario, 2017 [https://www. publichealthontario.ca/-/media/documents/annual-vaccine-safety-report-201 7.pdf?la=en].

36. Lankinen KPS, Kilpi T, Nohynek H, Makela P, Olin P. Vaccinovigilance in Europe--need for timeliness, standardization and resources. Bull World Health Organ. 2004;82(11):828-35.

37. Cashman PMK, Khandaker G, King C, Gold M, Durrheim D. Participantcentred active surveillance of adverse events following immunisation: a narrative review. Int Health. 2017;9(3):164-76.

38. World Health Organisation: Global advisory committee on vaccine safety, 23 December 2015. Weekly Epidermiological Record 2016, 91(3):24.

39. Yamoah POF. An assessment of the reporting pattern of adverse events folowing immunisations in VigiAccess. Global J Health Sci. 2018;10(11):46-56.

\section{Publisher's Note}

Springer Nature remains neutral with regard to jurisdictional claims in published maps and institutional affiliations.

\section{Ready to submit your research? Choose BMC and benefit from:}

- fast, convenient online submission

- thorough peer review by experienced researchers in your field

- rapid publication on acceptance

- support for research data, including large and complex data types

- gold Open Access which fosters wider collaboration and increased citations

- maximum visibility for your research: over $100 \mathrm{M}$ website views per year

At BMC, research is always in progress.

Learn more biomedcentral.com/submissions 